\title{
Preventing a feature-positive effect in pigeons
}

\author{
ULRIKE LINDENBLATT and JUAN D. DELIUS \\ Ruhr-Universität Bochum, Federal Republic of Germany
}

The feature-positive effect (FPE) is a widespread and robust phenomenon in the context of discrimination learning. It refers to the fact that a distinctive feature associated with a stimulus that is reinforced leads to efficient discrimination learning, whereas the same feature associated with the nonreinforced stimulus inhibits discrimination learning. Two experiments with pigeons showed that the FPE also occurs with a simultaneous discrimination paradigm involving brief discrete trials and no intertrial intervals. A pretraining treatment unexpectedly prevented the expression of the FPE in this discrimination task. The pretraining consisted of having pigeons discriminate the feature/nonfeature visual shapes from a plain background disc. Rewarding responses to the shapes, or alternatively to the blank disc, had the same FPE-preventing effect. A reversal of a feature/nonfeature stimulus discrimination led to an analogous erasure of the FPE. The results are discussed in terms of the concurrence or interference between the various associations that the subjects formed on the basis of the different stimulusreward correlations they experienced in the different phases of the experiments.

The feature-positive effect (FPE) was discovered by Jenkins and Sainsbury (1969). With an instrumental successive go/no-go paradigm including intertrial intervals (ITIs), they trained two groups of pigeons to discriminate two visual shapes displayed on a response key under two different conditions. The two shapes differed only by the presence or absence of a particular detail, a so-called feature. When the shape bearing this distinguishing element was the positive (reinforced) pattern and the negative (nonreinforced) was the plain shape (featurepositive condition $=$ FP condition), the pigeons learned the discrimination rapidly. When the reverse condition applied, that is, when the feature-bearing shape was the negative (nonreinforced) stimulus and the plain shape was the positive stimulus (feature-negative condition = FN condition), the pigeons showed little learning. Barring the fact that it is somewhat difficult to predict what will constitute a feature, the FPE has since been found in pigeons to be a robust effect resistant to a number of procedural variations (see Nallan, Miller, McCoy, 
Taylor, \& Serwatka, 1984, for a brief review). It has also been consistently obtained in several species other than the pigeon (e.g., rats: Halgren, 1974; Reberg \& Leclerc, 1977; cats: Diamond, Goldberg, \& Neff, 1962; monkeys: McCoy \& Yanko, 1983; Pace, McCoy, \& Nallan, 1980; humans: Nallan et al., 1986; Newman, Wolff, \& Hearst, 1980; Sainsbury, 1972).

Hearst $(1978,1984)$ interpreted the FPE in terms of a tendency of organisms to approach stimuli, or rather stimulus elements, that are predictive of reward (sign tracking). The pair of stimuli used in FPE experiments can be conceived as consisting of two elements. One is an element common to both stimuli $(A)$, and is in fact by itself one of the stimuli. The other is a feature $(X)$ that combined with the common element constitutes the alternative stimulus $(A X)$. In the FP condition the feature element $X$ is a perfect predictor of reward (100\%), whereas the common element $A$ is associated with reward on only $50 \%$ of the trials. This promotes responding to the feature stimulus, yielding good discriminative performance. In the FN condition, the common element $A$ is again associated with reward in $50 \%$ of the trials, but the feature $X$ now is only a perfect predictor of the absence of reward. In this situation there is nothing that promotes differential approach to the two stimuli $X$ and $A X$, and thus discrimination remains poor.

In the course of an abortive attempt to demonstrate a Stroop effect (Stroop, 1935) in pigeons, we incidentally found (a) that an FPE occurs with a simultaneous discrete-trial discrimination paradigm not involving ITIs and (b) that a particular kind of discriminative pretraining can prevent the occurrence of the FPE. The following experiments were designed to confirm and analyze these preliminary findings.

\section{EXPERIMENT 1}

In this experiment all pigeons learned to discriminate a feature stimulus $A X$ from a featureless stimulus $A$ either under the FP or the FN condition. Some pigeons, however, first learned to discriminate both the stimuli $A$ and $A X$ from a diffusely illuminated key.

\section{METHOD}

\section{Subjects and apparatus}

Twenty-four adult homing pigeons (Columbia livia) of local origin with no previous experimental experience were used. They were kept in individual cages $(40 \times 45 \times 40 \mathrm{~cm})$ in a well-ventilated, brightly lit (12L:12D) animal 
room with free access to water. The pigeons were food-deprived to $80 \%$ of their normal weight and maintained at that level throughout. A two-key Skinner box of conventional design and modular digital programming equipment were employed. Visual stimuli were back-projected onto the 25-mm diameter response keys with the aid of two multichannel microprojectors. Five stimuli were employed: a blank diffusely illuminated key, a circle, a nosed circle, a bar, and a gapped bar. The shapes, one to a key, appeared as black patterns on the bright background of the keys (Figure 1). With an autoshaping procedure, the pigeons were trained to peck the blank diffusely illuminated keys of a Skinner box for food reinforcement.

\section{Procedure}

The discrimination paradigm was of an operant simultaneous discretetrial type without ITIs. A trial began with the relevant pair of stimuli being projected on the keys. When the pigeon pecked the key bearing the stimulus defined as positive, the stimuli were turned off and food was offered for $1.5 \mathrm{~s}$. The next trial began immediately afterwards, the left-key/right-key
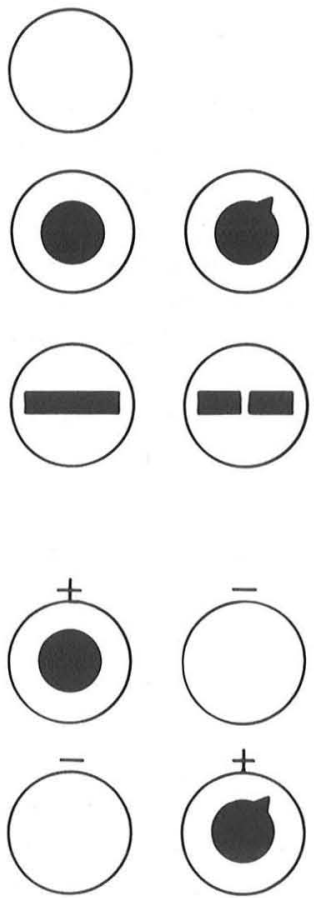

Figure 1. Stimuli employed: blank key, plain circle, nosed circle, plain bar, and gapped bar (bottom: examples of stimulus configurations used during positive pretraining: circles rewarded, blank key unrewarded) 
positions of the positive and negative stimuli being determined by a quasirandom sequence (Gellermann, 1933). If the pigeon responded to the negative stimulus, the stimuli and the houselight were turned off for $4 \mathrm{~s}$. The next trial began immediately afterward, but in this case the same stimuli were used, thus instituting a correction procedure that terminated only when the animal had responded correctly. The counters that recorded the correct and incorrect responses, however, disregarded the responses issued during correction trials. Daily sessions consisted of 100 trials, not counting correction trials.

The 24 pigeons were randomly divided into three groups of 8 pigeons each. Two of these groups, the pretrained groups, first learned to discriminate the circle patterns (both the plain circle and the nosed circle) from the blank illuminated key. For one group, the circles were the positive stimuli and the blank key was the negative stimulus; for the other group, the conditions were reversed. Pretraining consisted of six sessions. In 50 trials the plain shape and in the other $\mathbf{5 0}$ trials the feature stimulus shape appeared paired with a blank key stimulus. The two kinds of trials followed each other according to Gellermann sequences.

When pretraining was completed, all three groups (including the nonpretrained) learned to discriminate between the plain and the nosed circle for 10 sessions under the same conditions as described above. Within each group of 8 pigeons, one subgroup of 4 pigeons learned the discrimination under the FP condition and another subgroup of 4 pigeons learned the discrimination under the FN condition.

After this first phase, the 24 pigeons were reallocated to three new groups of 8 pigeons each. This was done randomly with the restriction that the previously nonpretrained pigeons were now all placed in the groups receiving pretraining. Exactly the same procedures as described above were followed in the second phase with the exception that instead of the circles, a bar and a gapped bar were used as featureless and featured stimuli.

\section{RESULTS}

The discriminative performance of the pigeons was assessed by calculating the mean percentage of correct trials (with standard errors) session by session across the 4 subjects of each of the six subgroups. These data are shown in Figure 2 for the positive pretrained and the nompretrained subgroups, and in Figure 3 for the negative pretrained, and for comparison's sake, for the nonpretrained subgroups.

The pretrained groups learned their initial discriminations quickly and to a high asymptote. There were marked differences between the performances of the nonpretrained groups and the pretrained groups regarding the discrimination of the featured and nonfeatured stimuli. The nonpretrained groups showed a clearcut FPE, that is, the FP subgroups learned the discrimination quickly and to a high standard, whereas the FN subgroups performed poorly. Both pre- 

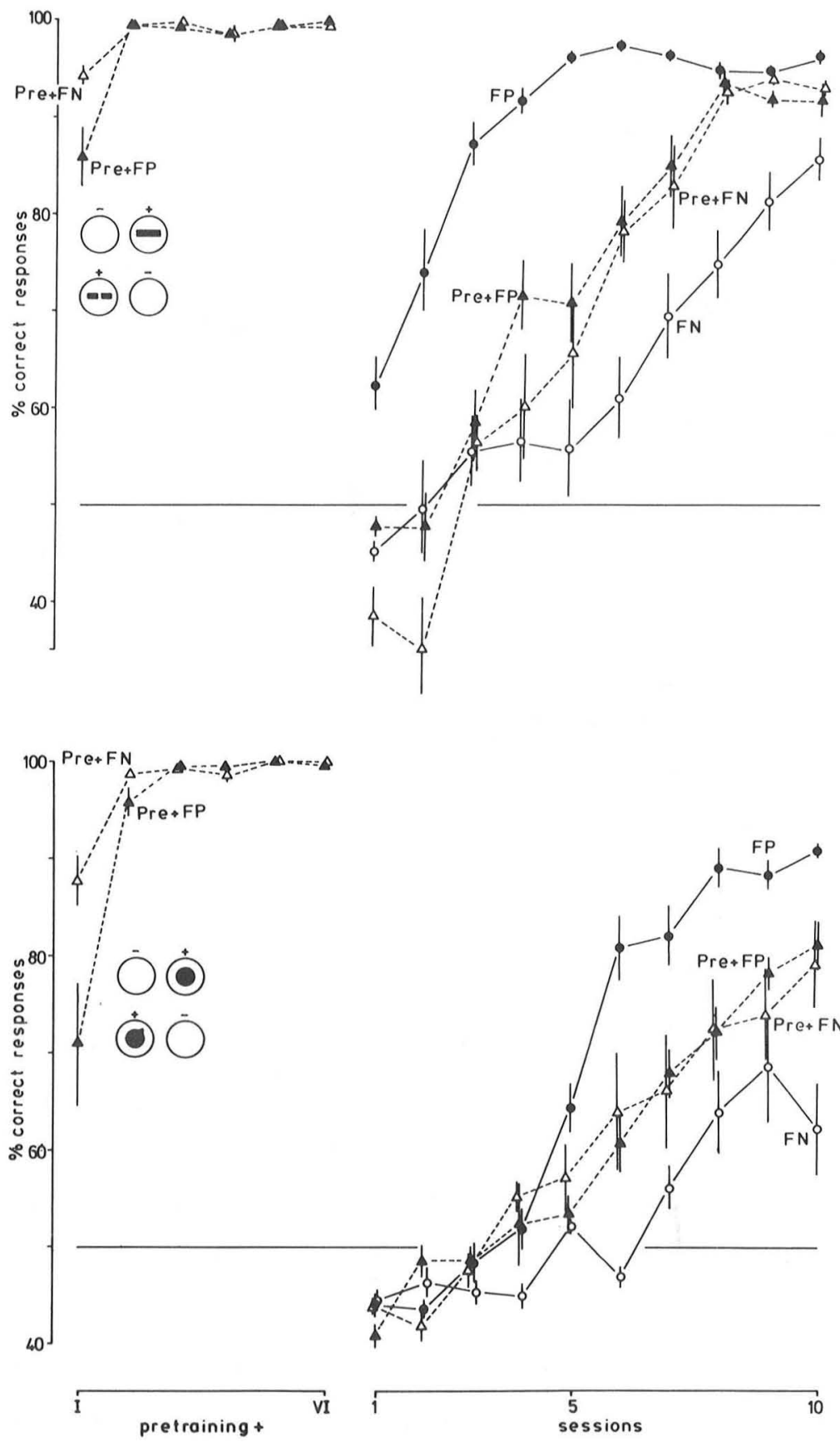

Figure 2. Mean discrimination learning curves (with standard errors) of the two nonpretrained and two positively pretrained subgroups of Experiment 1, both phases (circles and bars); Pre+FP = pattern positive pretrained, prospective feature positive; Pre $+\mathrm{FN}=$ pattern positive pretrained, pro- 

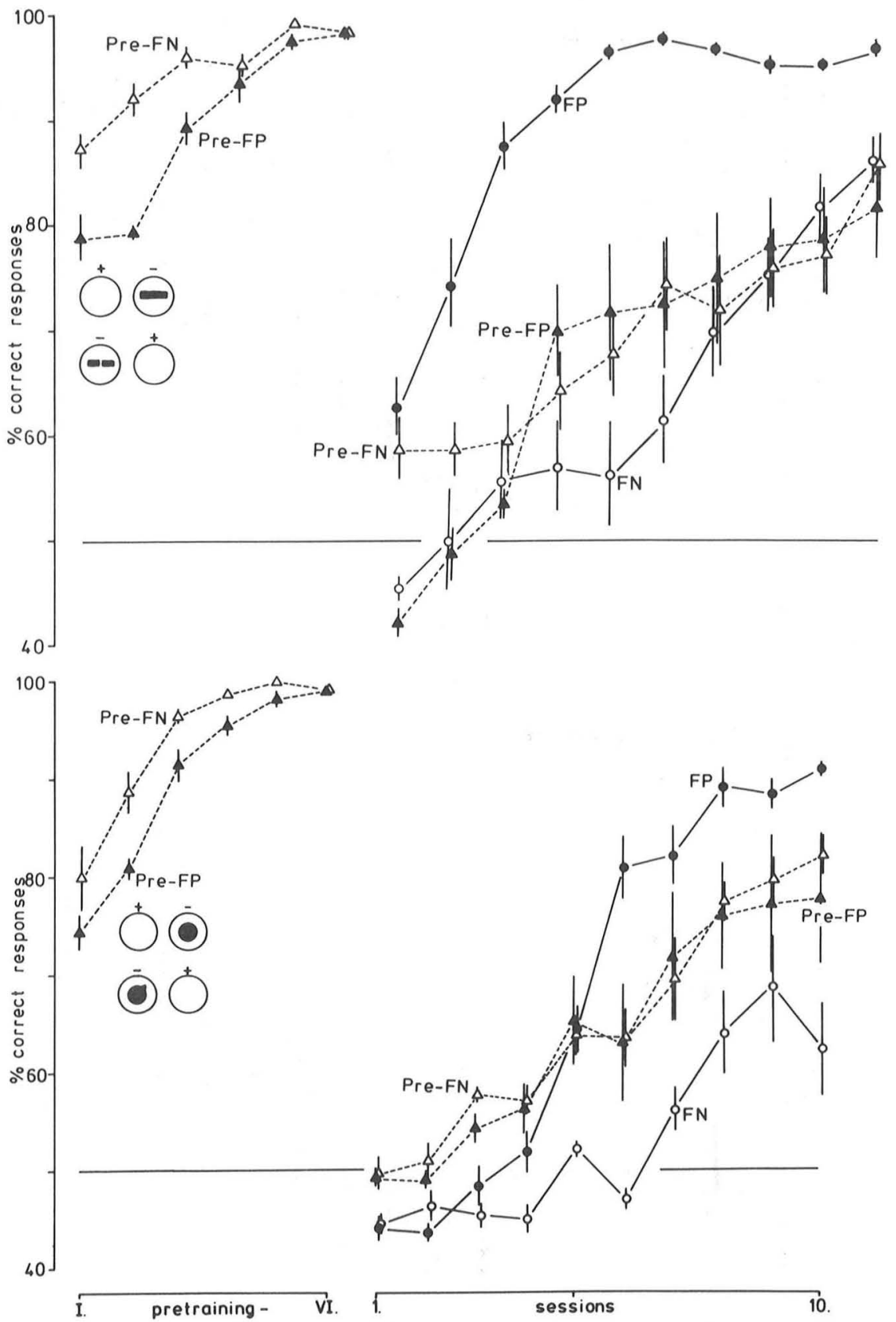

Figure 3. Mean discrimination learning curves (with standard errors) of the two nonpretrained (same as in Figure 2) and two negatively pretrained subgroups of Experiment 1, both phases (circles and bars); Pre-FP = pattern negative pretrained, prospective feature positive; Pre-FN = pattern negative pretrained, prospective feature negative; $F P=$ feature positive; $F N=$ feature negative 
trained groups yielded markedly reduced or even no performance differences between the FP and FN subgroups, their learning curves falling between those of the nonpretrained subgroups.

For the first phase (circle discrimination), an analysis of variance (Kirk, 1968) yielded a significant difference between the nonpretrained FP and FN subgroups, $F=11.467$, $d f 1 / 6, p<0.05$. All other treatment comparisons did not yield significant effects. In particular, there were no significant differences between the pretrained FP and FN subgroups. For the second phase (bar discrimination), a significant difference emerged again between the nonpretrained FP and FN subgroups, $F=14.969$, df $1 / 6, p<0.01$, but also between the nonpretrained FP subgroup and both pretrained FP subgroups, $F=10.322$ and $F=7.016$, df $1 / 6, p<0.05$. The latter results indicate that pretraining in this second phase had a larger inhibitory proactive effect on the discriminative performance of the FP subgroups than it had a proactive enhancing influence on the performance of the FN subgroups.

\section{DISCUSSION}

The FPE shown by our nonpretrained pigeons was somewhat weaker than that reported by Jenkins and Sainsbury $(1969,1970)$ employing, among others, one of the stimulus pairs used here. It is known, though, that the successive discrimination procedure they used is generally more difficult than the simultaneous procedure that we employed (Mackintosh, 1974). Nallan et al. (1984) nevertheless obtained a strong effect with a simultaneous paradigm but using quite different stimuli and no correction procedure. Hearst (1984) reported that shorter ITIs lead to a relatively better performance of FN subjects and thus to a weaker FPE. Our procedure involved no ITIs at all, and this might have improved the performance of our FN subjects. Nallan, Pace, McCoy, and Zentall (1979), however, found ITI duration to be an unimportant variable. Rather, they describe that the learning of FN subjects improved with longer trial durations. Our trials, however, were comparatively brief, as they lasted only as long as it took the pigeons to respond when the stimuli came on, less than $1 \mathrm{~s}$ on average.

The main result of this experiment was that the FPE shown by unpretrained pigeons was effectively prevented by the two varieties of pretraining that we employed, thus confirming our preliminary findings. The FPE-preventing effect of pretraining cannot be due to a general learning-set effect. The FPE shown by the nonpretrained group in the second phase (bars) was at least as marked as that evinced by the equivalent group during the first phase (circles). However, all 
the pigeons belonging to the nonpretrained group of the second phase had experienced discrimination learning during the first phase and, in fact, had also all been exposed to the pretraining corresponding to that phase. Thus, it cannot be that any discriminative pretraining will abolish the FPE.

It is thus reasonable to assume that the FPE prevention is due to the specific pretraining employed. The feature and the nonfeature stimuli first had to be discriminated against an illuminated key, the common element of all stimuli. The feature $X$ was associated with reward in only half of the trials, whereas the common, plain shape $A$ was maximally correlated with reward. The plain key exhibited zero correlation with reward. These correlations apply to both types of pretraining although the signs of the nonzero correlations were opposite: positive when responses to the shapes $(A$ and $A X)$ were rewarded, and negative when they were not. Either way, relative to the common, plain shape $A$, the feature $X$ was devalued as a predictor of reinforcement in the course of pretraining. Because $X$ is essential for the development of the FPE in nonpretrained animals, its devaluation could be expected to decrease the magnitude of the FPE.

This account serves well also when the positive contingency pretraining is considered in more detail. The stronger association of $A$ than of $X$ with reward, set up in the pigeons by this pretraining, promotes future responding to $A$ rather than to $X$, or by implication, to $A X$. During the FP training that follows, this opposes the reverse tendency that normally facilitates discrimination. Pretraining accordingly has a deleterious effect. In the $\mathrm{FN}$ situation where responding to $A$ and not to $A X$ is demanded, against the normal tendency, the pretraining has a facilitatory effect. Overall, the FPE is weakened by the positive contingency pretraining.

Why the negative contingency pretraining has the same FPEpreventing effect is less apparent. The stronger negative association of $A$ than of $X$ with reward, set up by this pretraining, should if anything facilitate subsequent responding to $X$ and hence to $A X$, rather than to $A$, thus magnifying the FPE. But it obviously does not do so; it decreases the FPE. An explanation might be provided by the socalled overtraining reversal effect (ORE). The ORE refers to the fact that strongly learned stimulus-reward correlations of a given sign can later be "converted" by subjects so that they facilitate associations of the opposite sign (Sutherland \& Mackintosh, 1971). An ORE would account for the FPE-preventing effect of the negative contingency pretraining by making it effectively equivalent to the positive contingency pretraining. 
Because the ORE is less than a universal effect (Mackintosh, 1974), this experiment examined whether it applies to the present context. A further goal was to reconfirm the FPE-preventing effect of the pretraining.

\section{METHOD}

\section{Subjects and apparatus}

Sixteen naive pigeons were used. The apparatus and stimuli were the same as in Experiment 1.

\section{Procedure}

The pigeons were first trained to key-peck and then arbitrarily allocated to two groups of 8 birds. The discrimination learning procedures were the same as in the previous experiment. One group was pretrained for six sessions, with responses to the circle/nosed circle rewarded and with responses to the blank key unrewarded. To minimize the number of subjects, and because both kinds of pretraining had identical effects in the previous experiment, only one pretrained group was run. The other group was not pretrained. Both groups, pretrained and nonpretrained, then discriminated the circle and nosed circle for six sessions, half of each group under FP conditions, the other half under $\mathrm{FN}$ conditions. The reinforcement conditions were then reversed for all pigeons, the former FN subgroups were now exposed to the FP condition and vice versa. After six reversal learning sessions, the pigeons were randomly reassigned to two groups of 8 for the second phase. The same procedure was followed except that the bar/gapped bar shapes were used as stimuli.

\section{RESULTS}

The data were treated in the same way as those of Experiment 1. Because both phases (circles and bars) again yielded quite similar results, means over both phases were calculated; these are shown in Figure 4. The pigeons' performance during the initial six sessions of each phase is equivalent to that shown by the pigeons in Experiment 1. Overall, the nonpretrained groups again yielded an FPE. Pretraining prevented an FPE in the circle/nosed circle discrimination and strongly attenuated it in the bar/gapped bar discrimination.

After the reversal of the reinforcement conditions during the last part of each phase, the nonpretrained pigeons failed to evince an FPE. The current FN subgroups (former FP subgroups) actually performed slightly better than the current FP (former FN) subgroups. 
The pretrained subgroups' performance was closely similar to that of the nonpretrained FP $\rightarrow$ FN subgroups (Figure 4). Generally the learning curves of all subgroups clustered closely together during the reversal discrimination section.

Analyses of variance (Kirk, 1968) showed that the nonpretrained pigeons in the circle/nosed circle discrimination yielded a significant FPE, $F=6.540$, df $1 / 6, p<0.05$. The difference between the nonpretrained FP and FN subgroups in the bar/gapped bar discrimination just failed to be significant. This was due to an untypically good performance of a single subject in the FN subgroup. The other treat-

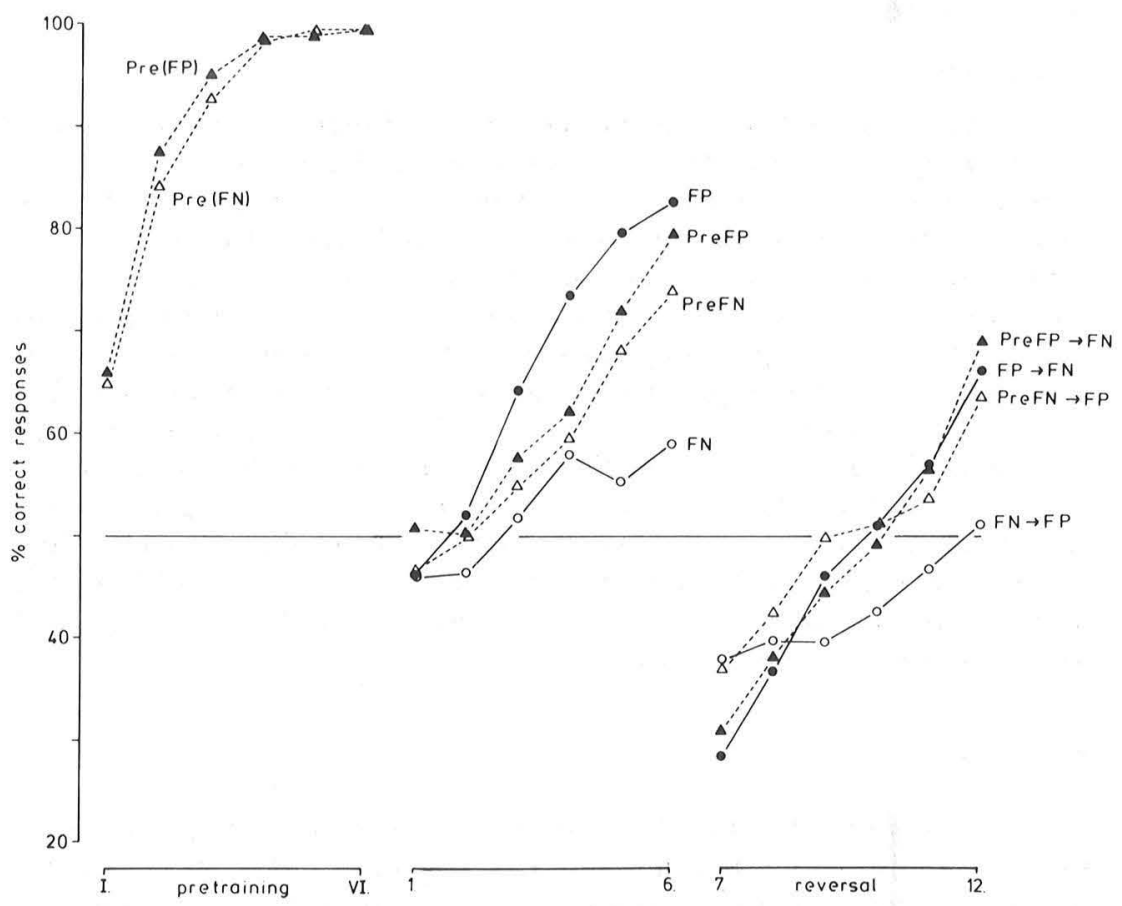

Figure 4. Overall mean discrimination learning curves of the four subgroups of Experiment 2 involving a reversal (results of the circle and bar phases have been combined); Pre(FP) = pretrained, prospective feature positive; $\operatorname{Pre}(\mathrm{FN})=$ pretrained, prospective feature negative; $\mathrm{PreFP}=$ pretrained feature positive; PreFN = pretrained feature negative; $\mathrm{FP}=$ feature positive; $\mathrm{FN}=$ feature negative; $\mathrm{FP} \rightarrow \mathrm{FN}=$ feature positive, reversed to feature negative; $\mathrm{FN} \rightarrow \mathrm{FP}=$ feature negative, reversed to feature positive; $\mathrm{PreFP} \rightarrow \mathrm{FN}=$ pretrained feature positive, reversed to feature negative; PreFN $\rightarrow \mathrm{FP}=$ pretrained feature negative, reversed to feature positive 
ment comparisons were nonsignificant, in particular all those concerning the reversal phase.

\section{DISCUSSION}

The performance of the pigeons that did not receive pretraining is considered first. The results of the first phases generally confirm the occurrence of an FPE. Upon reversal, however, the same pigeons evinced no FPE, but rather showed a tendency toward a featurenegative effect (FNE). That the FPE is absent during reversal learning and may even reverse into an FNE has also been reported for humans by Hearst (1984). The same review, however, reports an experiment on pigeons that yielded a clear FPE in the reversal phase. But in that experiment the training phase of the original FP group was markedly shorter than that of the original FN group. In the light of the argument developed below, this point may be significant for the presence of the FPE during reversal. In any case, Pace et al. (1980) report a clearcut weakening of the FPE after reversal for both pigeons and monkeys.

It will be recalled that the ORE refers to the fact that whereas learning a discrimination to a low criterion retards the acquisition of the reversal task (simple reversal effect, SRE), learning it to a high criterion actually facilitates it (Reid, 1953; Richman \& Coussens, 1970). We assume that our FN pigeons learned something about the negative correlation between the feature $X$ and reward even though this was weakly expressed in performance. Hearst (1984) marshalls evidence that FN pigeons do, in fact, partially learn about the negative correlation between feature and reward in spite of their poor discrimination performance. When confronted with the FP contingency, our subjects had first to unlearn that negative association and were thus slowed in the acquisition of the new positive correlation between $X$ and reward (SRE). Conversely, we assume that as reflected by their performance, our FP subjects learned very thoroughly about the positive correlation between $X$ and reward during the initial discrimination. This strong association is then easily inverted when faced with the negative correlation during reversal, thus favoring the learning of the new FN discrimination (ORE).

The positive pretraining in this experiment had the same effect as in Experiment 1. It depressed somewhat the acquisition of the original FP task and markedly facilitated that of the original FN task, thus preventing the emergence of an FPE and confirming the basic phenomenon. Upon reversal, the pretrained group also showed neither an FPE nor an FNE, in accordance with predictions that can be directly derived from the argument presented above. 


\section{GENERAL DISGUSSION}

Having shown in Experiment 2 that a straight discrimination reversal generates results that imply the operation of an ORE in an FPE context, we can now return to the explanation of the FPEpreventing effect of the negative contingency pretraining observed in Experiment 1. It can now be surmised with some confidence that the strong negative association between the common element $A$ and reward that was built up during the corresponding pretraining of Experiment 1 could indeed have had, thanks to the inverting of an ORE, an FPE-suppressing effect analogous to that produced by the positive association of the converse type of pretraining. Thus, essentially the same feature $X$ devaluation account that was offered for the FPE-preventing effect of the positive pretraining can now be applied to the negative one.

As to how the somewhat counterintuitive ORE comes about, we favor the explanation proposed by Sutherland and Mackintosh (1971). According to their theory, discrimination learning involves two stages. At a perceptual stage, stimulus-reinforcement correlations have, regardless of their sign, an equivalent attention-enhancing effect. At a response stage, the signs of the correlations lead to opposite effects, eliciting either approach or avoidance. Enhanced attention to a stimulus, or rather to a stimulus element (the common element $A$ in our case) built up during earlier training with whatever correlation sign, can later promote the learning of either approach or avoidance responses to it. Pace et al. (1980) put forward a similar attentional account to explain their result, showing that upon reversal the FPE evinced by monkeys and pigeons was weakened.

Attentional mechanisms are not foreign to FPE explanations, inasmuch as the definition of its basic element, the feature, calls for an attentionlike process. To be effective as a feature, a stimulus detail must "stand out" perceptually as an element of its own, as a figure out of the background, as $X$ out of $A X$. It must be able to "catch" the subject's attention. The capacity of certain stimulus details to do so must reflect the properties of specific perceptual filters, which are present before the FPE experiments begin. Regardless of whether they arose through phylogeny or ontogeny, they are likely to be related to the perceptually essential function of distinguishing objects from background. There is evidence that the pecking of stimuli displayed on operant chamber keys is closely related to food gathering (Delius, 1986). LaMon and Zeigler (1984) demonstrated that the gape amplitude of pigeon key pecks is a function of the diameter of grains offered as reward. Earlier, Jenkins and Sainsbury (1970; see also Nallan 
et al., 1984) reported that pigeons aim their pecks at the features in FP situations and avoid them in FN contexts. The FPE may thus relate to optimal foraging behavior, to an efficient identification of grains against a background of dirt and grit (Bond, 1983). Thus, what a pigeon considers a feature may coincide with what it perceives as cues distinctive of food objects vis-à-vis background substrates.

\section{Notes}

This research was supported by the Deutsche Forschungsgemeinschaft through its Sonderforschungsbereich 114. While preparing this report, U. Lindenblatt received a grant from the Graduiertenförderung (NRW). We thank D. Hagenkötter, A. Lohmann, and J. Delius for their assistance.

Requests for offprints should be addressed to Juan D. Delius, Allgemeine Psychologie, Universität Konstanz, D7750 Konstanz, FRG. Received for publication August 6, 1986; revision received December 12, 1986.

\section{References}

Bond, A. B. (1983). Visual search and selection of natural stimuli in the pigeon: The attention threshold hypothesis. Journal of Experimental Psychology: Animal Behavior Processes, 9, 292-306.

Delius, J. D. (1986). The peck of the pigeon: Free for all. In C. F. Lowe, M. Richelle, D. E. Blackman, \& C. M. Bradshaw (Eds.), Behavior analysis and contemporary psychology (pp. 53-81). Hillsdale, NJ: Erlbaum.

Diamond, I. T., Goldberg, J. M., \& Neff, W. D. (1962). Tonal discrimination after ablation of auditory cortex. Journal of Neurophysiology, 25, 223-235.

Gellermann, L. W. (1933). Chance orders of alternating stimuli in visual discrimination experiments. Journal of Genetic Psychology, 42, 206-208.

Halgren, C. R. (1974). Latent inhibition in rats: Associative or nonassociative? Journal of Comparative and Physiological Psychology, 86, 74-78.

Hearst, E. (1978). Stimulus relationships and feature selection in learning and behavior. In S. H. Hulse, H. Fowler, \& W. K. Honig (Eds.), Cognitive processes in animal behavior (pp. 51-88). Hillsdale, NJ: Erlbaum.

Hearst, E. (1984). Absence as information: Some implications for learning, performance, and representational processes. In H. L. Roitblat, T. G. Bever, \& S. Terrace (Eds.), Animal cognition (pp. 311-332). Hillsdale, NJ: Erlbaum.

Jenkins, H. M., \& Sainsbury, R. S. (1969). The development of stimulus control through differential reinforcement. In N. J. Mackintosh \& W. K. Honig (Eds.), Fundamental issues in associative learning (pp. 123-161). Halifax, NS: Dalhousie University Press.

Jenkins, H. M., \& Sainsbury, R. S. (1970). Discrimination learning with the distinctive feature on positive or negative trials. In D. J. Mostofsky (Ed.), Attention: Contemporary theory and analysis (pp. 239-273). New York: Appleton-Century-Crofts. 
Kirk, R. E. (1968). Experimental design: Procedures for the behavioral sciences. Belmont, CA: Brooks/Cole.

LaMon, B. C., \& Zeigler, H. P. (1984). Grasping in the pigeon (Columba livia): Stimulus control during conditioned and consummatory responses. Animal Learning and Behavior, 12, 223-231.

Mackintosh, N. J. (1974). The psychology of animal learning. London: Academic Press.

McCoy, D. F., \& Yanko, D. M. (1983). Feature-positive and feature-negative discrimination learning maintained by negative reinforcement in rhesus monkeys. Animal Learning and Behavior, 11, 460-464.

Nallan, G. B., Miller, J. S., McCoy, D. F., Taylor, R. T., \& Serwatka, J. (1984). Transfer effects in feature-positive and feature-negative learning by pigeons. American Journal of Psychology, 97, 509-518.

Nallan, G. B., Pace, G. M., McCoy, D. F., \& Zentall, T. R. (1979). Temporal parameters of the feature-positive effect. American Journal of Psychology, 92, 703-710.

Nallan, G. B., Sanders, R., Dykeman, C., Hughes, M., Rauth, M., McCann, S., \& Morrison-Nallan, K. (1986). Identity relation can serve as the distinguishing feature in feature-positive and feature-negative learning research. American Journal of Psychology, 99, 71-79.

Newman, J., Wolff, W. T., \& Hearst, E. (1980). The feature-positive effect in adult human subjects. Journal of Experimental Psychology: Human Learning and Memory, 6, 630-650.

Pace, G. M., McCoy, D. F., \& Nallan, G. B. (1980). Feature-positive and feature-negative learning in the Rhesus monkey and pigeon. American Journal of Psychology, 93, 409-427.

Reberg, D., \& Leclerc, R. (1977). A feature-positive effect in conditioned suppression. Animal Learning and Behavior, 5, 143-147.

Reid, L. S. (1953). The development of noncontinuity behavior through continuity learning. Journal of Experimental Psychology, 46, 107-112.

Richman, C. L., \& Coussens, W. (1970). Undertraining reversal effect in rats. Journal of Experimental Psychology, 86, 340-342.

Sainsbury, R. S. (1972). The "feature positive effects" and simultaneous discrimination learning. Journal of Experimental Child Psychology, 11, 347-356.

Stroop, J. R. (1935). Studies of interferences in serial verbal reactions. Journal of Experimental Psychology, 18, 643-662.

Sutherland, N. S., \& Mackintosh, N. J. (1971). Mechanisms of animal discrimination learning. New York: Academic Press. 